Research Article

\title{
Enzyme replacement therapy for Fabry disease: A systematic review and meta-analysis
}

\author{
Taciane Alegra ${ }^{1}$, Filippo Vairo ${ }^{1,2}$, Monica V. de Souza ${ }^{3}$, Bárbara C. Krug $^{3}$ and Ida V.D. Schwartz ${ }^{1,2,3,4}$ \\ ${ }^{1}$ Programa de Pós-Graduação em Genética e Biologia Molecular, \\ Universidade Federal do Rio Grande do Sul,Porto Alegre, RS, Brazil. \\ ${ }^{2}$ Serviço de Genética Médica, Hospital de Clínicas de Porto Alegre, Porto Alegre, RS, Brazil. \\ ${ }^{3}$ Programa de Pós-Graduação em Ciências Médicas, Universidade Federal do Rio Grande do Sul, \\ Porto Alegre, RS, Brazil. \\ ${ }^{4}$ Departamento de Genética, Universidade Federal do Rio Grande do Sul, Porto Alegre, RS, Brazil.
}

\begin{abstract}
The specific treatment available for Fabry disease (FD) is enzyme replacement therapy (ERT) with agalsidase alfa or beta. A systematic review and meta-analysis was conducted to assess the efficacy and safety of ERT for FD. Only double-blind, randomized clinical trials (RCTs) comparing agalsidase alfa or beta and placebo were included. ERT with either agalsidase alfa or beta was considered similar for the purposes of analysis. Ten RCTs were identified, which showed improvements in neuropathic pain, in heart abnormalities and in globotriaosylceramide (GL-3) levels. A meta-analysis showed increased odds for fever, rigors, development of IgG antibodies to agalsidase, and no significant association with development of hypertension or reduction in the QRS complex duration on electrocardiogram. The RCTs included in this comparison enrolled few patients, were highly heterogeneous, and were focused mainly on surrogate endpoints, limiting any conclusions as to the real effect of ERT for FD. The available evidence suggests that response to ERT is variable across patient subgroups and that agalsidase may slow progression of FD, with slight improvement of existing changes. Nevertheless, many uncertainties remain, and further studies are necessary.
\end{abstract}

Keywords: Fabry disease, Fabry disease/therapy, enzyme replacement therapy, alpha-Galactosidase.

\section{Introduction}

Fabry disease (FD) is an X-linked lysosomal storage disorder characterized by deficient activity of $\alpha$-galactosidase A ( $\alpha \mathrm{GAL}-\mathrm{A})$, one of the enzymes involved in glycosphingolipid catabolism. Consequently, patients exhibit progressive storage of globotriaosylceramide (GL-3) throughout the body, and particularly so in vascular endothelium. The incidence of FD hemizygosity is generally estimated as 1 in 40,000 to 50,000 males (Meikle et al., 1999; Desnick et al., 2001); however, recognition of atypical forms of the disease and neonatal screening (Spada et al., 2006) suggest that the actual figure may be much higher.

Unlike most lysosomal disorders, FD mostly afflicts young adults (Wraith, 2004). In the classic form of the disease, clinical manifestations begin at the age of 5. The most common findings include intermittent acroparesthesia

Send correspondence to Ida Vanessa Doederlein Schwartz. Serviço de Genética Médica, Hospital de Clínicas de Porto Alegre, Rua Ramiro Barcelos 2350, 90035-903 Porto Alegre, RS, Brazil. E-mail: ischwartz@ @cpa.ufrgs.br.
("Fabry crisis"), with episodes of acute pain lasting from hours to days; angiokeratomas; cornea verticillata; hypohydrosis or anhydrosis; heat, cold and exercise intolerance; mild proteinuria; and gastrointestinal disorders (Eng et al., 2007). Cardiac complications include left ventricular hypertrophy, heart valve disease, coronary artery disease, conduction abnormalities, heart failure, arrhythmias and acute myocardial infarction (Pieroni et al., 2006). Cerebrovascular disease is manifest as white matter lesions, paresthesias, vertigo, early stroke and transient ischemic attacks (Politei and Capizzano, 2006). Glomerular podocyte injury leads to proteinuria and hematuria, which are usually detected during adolescence. Organ and system involvement is progressive, and by the third to fifth decade of life most patients have developed severe kidney and heart disease (Eng et al., 2007). Progressive renal dysfunction eventually requires dialysis and renal transplantation, and is the main cause of death in FD (Desnick et al., 2001; Eng et al., 2007). Atypical forms follow an oligosymptomatic course, with involvement mostly confined to one system (renal disease, for instance). As FD is an X-linked condition, the phe- 
notype in females varies widely, ranging from complete absence of symptoms to severe disease (Wilcox et al., 2008; Pinto et al., 2010).

Supportive therapy of FD includes pain management with analgesics, anticonvulsants and non-steroidal antiinflammatory agents, treatment of cerebrovascular, cardiac and renal comorbidities with antiplatelet and antihypertensive agents, dialysis and, in end-stage renal disease, kidney transplantation. Specific treatment consists of enzyme replacement therapy (ERT) with recombinant $\alpha$ GAL-A (agalsidase). Two agalsidase products are currently available on the market, agalsidase alfa and agalsidase beta. Agalsidase alfa is manufactured by Shire Human Genetic Therapies (Cambridge, MA, USA) from human cell lines and administered every two weeks by intravenous infusion (over $40 \mathrm{~min}$ ) at a dose of $0.2 \mathrm{mg} / \mathrm{kg}$. Agalsidase beta is manufactured by Genzyme Corporation, which was recently acquired by Sanofi-Aventis (Paris, France), from $\mathrm{CHO}$ (Chinese hamster ovary) cells and administered every two weeks by intravenous infusion at a dose of $1 \mathrm{mg} / \mathrm{kg}$ at an initial rate of $0.25 \mathrm{mg} / \mathrm{min}$. Both products have been approved for use in the European Union since 2003, but only agalsidase beta has been cleared by the U.S. Food and Drug Administration (FDA) for use in the USA. In Brazil, agalsidase beta and agalsidase alfa received marketing authorization in 2006 and 2009 respectively.

As a multisystem disease requiring lifelong follow-up and clinical monitoring, FD has a substantial impact on healthcare systems, not only due to the high cost of ERT (approximately R $\$ 350,000.00$ per year per patient), but also due to the morbidity experienced by patients, usually during working age. The present study sought to evaluate, through a systematic review of the literature (SR) and meta-analysis, the efficacy and safety of ERT for treatment of FD.

\section{Methods}

In vitro studies suggest that agalsidase alfa and beta share very high biochemical and structural similarity and are indistinguishable in terms of functional effects. Both have identical amino acid sequences, specific activity and stability (Blom et al., 2003), and show only minor differences in glycosylation (Lee et al., 2003) and mannose6-phosphate receptor mediated cellular uptake (Sakuraba et al., 2006). The only head-to-head comparison of agalsidase alfa and beta, both given at a dose of $0.2 \mathrm{mg} / \mathrm{kg}$ biweekly (Vedder et al., 2007), revealed no clinically relevant difference between the enzymes. Therefore, for the purposes of this SR, agalsidase alfa and beta were considered similar pharmaceutical agents, and study results will be presented according to dose $(0.2 \mathrm{mg} / \mathrm{kg}$ for agalsidase alfa and 1 $\mathrm{mg} / \mathrm{kg}$ for agalsidase beta) rather than by agent.

\section{Systematic review}

The SR was carried out according to the guidelines of the Cochrane Collaboration (Higgins and Green, 2011). Two investigators searched the literature independently, identified articles of interest by scanning titles and abstracts when available, and, after a consensus meeting, compiled a single list of suitable articles, the full text of which was then read by two other investigators. The reference lists of selected articles were also assessed with a view to identification of other studies of interest that might not have been identified by the main search strategy. The methodological quality of the randomized clinical trials (RCTs) was assessed against a modified version of the Jadad scale (Woodroffe et al., 2005), which evaluates each study with regard to randomization, blinding and subject withdrawals and dropouts. Studies with a score of 0 to 2 are considered low quality, 3 to 4 as of moderate and 5 to 6 as of high quality.

The Embase, PUBMED/MEDLINE and LILACS databases were searched for articles published until July 30 , 2010 or March 30, 2011 respectively. According to predefined criteria, only double-blind RCTs comparing ERT to other therapies or placebo for treatment of FD were included in the review.

The search strategies were applied as follows in the PUBMED/MEDLINE: ((fabry disease OR fabry's disease) AND (agalsidase beta OR enzyme replacement therapy)) OR ((fabry disease OR fabry's disease) AND (agalsidase alfa OR enzyme replacement therapy)). In Embase the search strategy was: 'Fabry disease' / exp AND 'therapy' / exp; in the LILACS: Fabry [Words] and therapy [words]. "Human studies" and "reviews, meta-analysis, randomized controlled trials" were used as search limits in the PUBMED/MEDLINE and Embase respectively.

During the data extraction process, complementary information not available in the selected articles was also searched in clinical trial registries (http://www.clinicaltrials.gov, http://www.controlledtrials.com, and the Cochrane Register of Controlled Trials).

When measures of treatment effect were not directly expressed they were calculated from other available data, with WinPepi 10.7 software. Relative risk and difference between means were calculated for dichotomous and continuous data respectively, both with a $95 \%$ confidence interval (CI). When data were expressed with standard error (SE) measurements, the latter were converted to standard deviations (SD) by multiplying the SE by the square root of the number of patients $n(\mathrm{SD}=\mathrm{SE} \times \sqrt{ } \mathrm{n})$.

\section{Meta-analysis}

For continuous variables, within-study variances were estimated using a $t$ distribution. Meta-analyses were carried out using both fixed and random effects models, represented by the Peto (Bradburn et al., 2007) and DerSimonian-Laird (DerSimonian and Laird, 1986) methods respectively. The Peto method is a fixed effects model-based 
approach that has demonstrably superior statistical properties under scenarios with rare events (Bradburn et al., 2007). Statistical heterogeneity was tested using the Cochran $Q$ test and quantified using the $I^{2}$ index. $\mathrm{p}<0.05$ was considered significant evidence of treatment effect. For the $Q$ statistic, which is a test with relatively low power in most scenarios, a significance level of $\mathrm{p}<0.1$ was chosen. Statistical analyses were carried out in the Stata 11.1 package (Stata Corporation, College Station, TX, USA).

\section{Results}

A total of 547 studies were initially identified, but only 10 were double-blind, randomized, placebo-controlled trials of agalsidase alfa or beta. No studies comparing ERT to other therapies were found, and no additional information was obtained from the clinical trials registries.

Tables 1 and 2 provide an overview of the main characteristics of the studies included. Among these, five were classified as high quality (Moore et al., 2001, 2002; Schiffmann et al., 2001, 2006; Banikazemi et al., 2007;). Four others received a score of 3 for failing to describe the method of randomization (Eng et al., 2001; Thurberg et al., 2002, 2004; Hughes et al., 2008;). Only one RCT received a score of $<3$ points (Bierer et al., 2006).

\section{Agalsidase at $0.2 \mathrm{mg} / \mathrm{kg} / \mathrm{infusion}$}

This SR included five RCTs of agalsidase given at a dose of $0.2 \mathrm{mg} / \mathrm{kg} /$ infusion (Moore et al., 2001, 2002; Schiffmann et al., 2001, 2006; Hughes et al., 2008;) (Tables 1 and 2). The use of premedication was not reported by the authors.

A total of 41 subjects were included in these studies since the studies by Moore et al. (2001, 2002), Schiffmann et al. $(2001,2006)$ evaluated the same patient sample; they were all males, older than 18 years of age, with neuropathic pain. One patient, allocated in the placebo group, failed to complete all proposed stages after randomization in the cohort evaluated. The study by Hughes et al. (2008) conducted on the same sex/age group included subjects with evidence of left ventricular hypertrophy. This study did not report any patients lost to follow-up, but one patient from each group (drug/placebo) was excluded in two subgroup analyses (one patient from the myocardial GL-3 analysis due to the development of an arrhythmia during his baseline cardiac biopsy procedure, and one placebo from MRI left ventricular mass analysis due to claustrophobia).

\section{Agalsidase at $1 \mathrm{mg} / \mathrm{kg} / \mathrm{infusion}$}

This SR also included five double-blind, randomized, placebo-controlled trials of agalsidase given at a dose of 1 $\mathrm{mg} / \mathrm{kg} /$ infusion (Eng et al., 2001; Thurberg et al., 2002, 2004; Bierer et al., 2006; Banikazemi et al., 2007) (Tables 1 and 2). The patients were pretreated with acetaminophen or ibuprofen, and some with an antihistamine as well (Eng et al., 2001; Banikazemi et al., 2007).

In all, 146 patients took part in these studies, only 13 of whom were female. Three studies (Eng et al., 2001; Thurberg et al., 2002, 2004) evaluated the same patient sample; they had as criteria for inclusion: age $>16$ years, serum creatinine $<2.2 \mathrm{mg} / \mathrm{dL}$, and absence of kidney disease requiring renal replacement therapy (patients on dialysis or awaiting transplant). In one study (Banikazemi et al., 2007), the inclusion criteria were: age $>16$ years, evidence of mild to moderate renal failure, with serum creatinine $\geq 1.2 \mathrm{mg} / \mathrm{dL}$ and $<3 \mathrm{mg} / \mathrm{dL}$ or calculated creatinine clearance $<1.33 \mathrm{~mL} / \mathrm{s}$; patients were excluded in case of kidney disease requiring renal replacement therapy or history of transient ischemic attack or ischemic stroke, unstable an-

Table 1 - Overview of the double-blind, randomized, placebo-controlled trials of enzyme replacement therapy with agalsidase for Fabry disease.

\begin{tabular}{|c|c|c|c|c|c|}
\hline Authors & $\mathrm{n}(\mathrm{M}: \mathrm{F})$ & $\begin{array}{l}\text { Intervention group dose } \\
\text { of agalsidase (n) }\end{array}$ & Age in years mean (range) & $\begin{array}{c}\text { Duration of the } \\
\text { double-blind } \\
\text { phase of study } \\
\text { (months) }\end{array}$ & $\begin{array}{c}\text { Quality } \\
\text { score (modi- } \\
\text { fied Jadad } \\
\text { scale) }^{\mathrm{e}}\end{array}$ \\
\hline Eng et al. $(2001)^{\mathrm{a}}$ & $58(56: 2)$ & $1 \mathrm{mg} / \mathrm{kg}(\mathrm{n}=29)$ & Intervention $=32.0 /$ Placebo $=28.4(17-61)$ & 5 & 5 \\
\hline Thurberg et al. $(2002)^{\mathrm{a}}$ & $58(56: 2)$ & $1 \mathrm{mg} / \mathrm{kg}(\mathrm{n}=29)$ & Intervention $=32.0 /$ Placebo $=28.4(17-61)$ & 5 & 5 \\
\hline Thurberg et al. $(2004)^{\mathrm{a}}$ & $58(56: 2)$ & $1 \mathrm{mg} / \mathrm{kg}(\mathrm{n}=29)$ & Intervention $=32.0 /$ Placebo $=28.4(17-61)$ & 5 & 5 \\
\hline Schiffmann et al. $(2001)^{\mathrm{b}}$ & $26(26: 0)$ & $0.2 \mathrm{mg} / \mathrm{kg}(\mathrm{n}=14)$ & Intervention $=34.0 /$ Placebo $=34.4^{\mathrm{c}}$ & 6 & 3 \\
\hline Moore et al. $(2001)^{\mathrm{b}}$ & $26(26: 0)$ & $0.2 \mathrm{mg} / \mathrm{kg}(\mathrm{n}=14)$ & Intervention $=34.0 /$ Placebo $=34.4^{\mathrm{c}}$ & 6 & 3 \\
\hline Moore et $a l .(2002)^{b}$ & $26(26: 0)$ & $0.2 \mathrm{mg} / \mathrm{kg}(\mathrm{n}=14)$ & Intervention $=34.0 /$ Placebo $=34.4^{\mathrm{c}}$ & 6 & 3 \\
\hline Schiffmann et al. $(2006)^{\mathrm{b}}$ & $26(26: 0)$ & $0.2 \mathrm{mg} / \mathrm{kg}(\mathrm{n}=14)$ & Intervention $=34.0 /$ Placebo $=34.4^{\mathrm{c}}$ & 6 & 3 \\
\hline Bierer et al. (2006) & $6(5: 1)$ & $1 \mathrm{mg} / \mathrm{kg}(\mathrm{n}=4)$ & Males $=35 /$ Females $=42(20-47)$ & 18 & 1 \\
\hline Hughes et al. (2008) & $15(15: 0)$ & $0.2 \mathrm{mg} / \mathrm{kg}(\mathrm{n}=7)$ & Intervention $=37.1 /$ Placebo $=37.3(23-50)$ & 6 & \\
\hline
\end{tabular}

${ }^{a}$ These studies included the same patient sample. ${ }^{\mathrm{b}}$ These studies included the same patient sample. ${ }^{\mathrm{c}}$ Range not reported. ${ }^{\mathrm{d}}$ Multicentric study. ${ }^{\mathrm{e}}$ The methodological quality was assessed against a modified version of the Jadad scale (Woodroffe et al., 2005). Studies with a score of 0 to 2 are considered low quality, 3 to 4 are considered moderate quality and 5 to 6 are considered high quality. 
Table 2 - Summary of the efficacy results of enzyme replacement therapy with agalsidase for Fabry disease

\begin{tabular}{|c|c|c|c|c|c|}
\hline Outcomes & $\begin{array}{l}\text { Eng et al. (2001), } \\
\text { Thurberg et al. (2002), } \\
\text { Thurberg et al. (2004) }\end{array}$ & $\begin{array}{l}\text { Schiffmann et al. (2001), } \\
\text { Moore et al. (2001), } \\
\text { Moore et al. (2002), } \\
\text { Schiffmann et al. (2006) }\end{array}$ & $\begin{array}{c}\text { Bierer } \\
\text { et al. }(2006)\end{array}$ & $\begin{array}{l}\text { Banikazemi } \\
\text { et al. (2007) }\end{array}$ & $\begin{array}{l}\text { Hughes } \\
\text { et al. }(2008)\end{array}$ \\
\hline Blood pressure & - & - & NS & - & NS \\
\hline Cardiovascular performance during exercise & - & - & NS & - & - \\
\hline Cerebral blood flow (resting) & - & $\downarrow$ & - & - & - \\
\hline Cerebral blood flow after visual stimulation & - & $\downarrow$ & - & - & - \\
\hline Death & - & - & - & NS & - \\
\hline ECG findings & NS & $\downarrow$ QRS & - & - & NS \\
\hline Echocardiographic findings & NS & - & - & - & NS \\
\hline GL-3 clearance in plasma & $\downarrow$ & $\downarrow$ & - & - & $\downarrow$ \\
\hline GL-3 clearance in urinary sediment & $\downarrow$ & NS & - & - & $\downarrow$ \\
\hline GL-3 content in heart & $\downarrow$ & - & - & - & NS \\
\hline GL-3 content in kidney & $\downarrow$ & NS & - & - & - \\
\hline GL-3 content in skin & $\downarrow$ & - & - & - & - \\
\hline eGFR & NS & NS & - & NS & NS \\
\hline LVM (MRI) & - & - & - & - & $\downarrow$ \\
\hline $\begin{array}{l}\text { Intraepidermal nerve fiber density and ther- } \\
\text { mal threshold }\end{array}$ & - & NS & - & - & - \\
\hline Neuropathic pain & NS* & $\downarrow * *$ & - & - & - \\
\hline Pain-related QoL & - & $\uparrow$ & - & - & - \\
\hline QoL (SF-36 questionnaire) & NS & - & - & - & - \\
\hline Proteinuria & - & NS & - & NS & - \\
\hline Time to first clinical event \# & - & - & - & NS & - \\
\hline
\end{tabular}

$\uparrow=$ improvement; $\downarrow$ = decrease; - = not evaluated; \# Clinical events: renal, cardiac, cerebrovascular or death; * Pain was assessed by the McGill Questionnaire; ** Pain was assessed by the Brief Pain Inventory; eGFR, estimated glomerular filtration rate; GL-3, globotriaosylceramide; NS, not significant; QoL, quality of life; LVM, left ventricular mass; MRI = magnetic resonance imaging.

gina or acute myocardial infarction less than 3 months prior to enrollment. The study by Bierer et al. (2006) mentioned no criteria for inclusion other than a diagnosis of FD. Only one study had withdrawals or dropouts (Banikazemi et al., 2007), where three patients left the trial to receive commercial therapy (two in the agalsidase and one in the placebo group), another three patients in the agalsidase group withdrew due to suspected or confirmed IgE-mediated reactions, two (one in each group) left due to unknown reasons ("voluntary", after the occurrence of a clinically relevant event), and three died (two patients, from the agalsidase group, died of pulmonary emboli and sudden cardiac arrest, respectively; and one patient, from the placebo group, died of cardiac arrest one week after a stroke).

\section{Efficacy and safety assessment}

The data on efficacy assessment of ERT for FD is summarized in Table 2. This table describes the outcomes/endpoints evaluated in each trial.

Regarding safety, only one study evaluated adverse effects of ERT when given at a dose of $0.2 \mathrm{mg} / \mathrm{kg} /$ infusion (Schiffmann et al., 2001). Mild infusion-related events (most commonly rigors) were not found in the placebo group, but occurred in eight of 14 patients given agalsidase. Premedication with antihistamines and low-dose corticosteroids reduced these reactions, as did slower infusion rates during subsequent treatment sessions.

Two studies described the adverse effects of the administration of agalsidase at a dose of $1 \mathrm{mg} / \mathrm{kg} /$ infusion (Eng et al., 2001; Banikazemi et al., 2007). Fever and rigors were more common in the ERT group than with placebo (Eng et al., 2001; Banikazemi et al., 2007). Without statistical differences between the placebo and agalsidase group were reports on hypertension (Eng et al., 2001; Banikazemi et al., 2007;), headache (Eng et al., 2001), chills (Eng et al., 2001), FD-related pain (Eng et al., 2001), vomiting (Banikazemi et al., 2007), perceived temperature changes (Banikazemi et al., 2007), chest pain (Banikazemi et al., 2007) and fatigue (Banikazemi et al., 2007).

Severe treatment-related adverse effects were described in three out of 51 patients given agalsidase at a dose of $1 \mathrm{mg} / \mathrm{kg} /$ infusion, but none were so in the placebo group (Banikazemi et al., 2007). One patient developed severe hypotension, followed by detectable anti-agalsidase IgE 
antibodies in serum, one developed urticaria and glottis edema, and the third had urticaria, chills and fever (Banikazemi et al., 2007).

\section{Variables selected for meta-analysis}

Data extracted from the SR were pooled for metaanalysis. Some studies shared the same endpoints, but few reported absolute post-treatment measurements, measurement units, or the method used for deriving results. For instance, renal function outcomes (measured by creatinine or inulin clearance) were assessed in four studies (Eng et al., 2001; Schiffmann et al., 2001; Banikazemi et al., 2007; Hughes et al., 2008), but only one provided numerical values (Schiffmann et al., 2001), the other studies only reported it descriptively (function "remained stable"). Another variable affected by this issue was plasma, urinary and tissue clearance of GL-3, for which outcomes were alternatively presented as absolute values, relative percentage change, or change measured against a standardized score, making it impossible to pool results for statistical analysis. Therefore, only the following variables could be included in the meta-analysis: duration of QRS complex (the portion of the electrocardiogram comprising the Q, R and $\mathrm{S}$ waves, together representing ventricular depolarization), and the incidence of fever, rigors, hypertension and development of $\operatorname{IgG}$ antibodies to agalsidase as adverse events.

Regarding QRS duration, as sample size was small and one study (Hughes et al., 2008) did not report SDs, we calculated the summary effect for each potential alternative $\mathrm{SD}$ and found that, regardless of the estimated SD, results were not statistically significant (Figure 1).

Few adverse events were reported in more than one study: hypertension, fever, rigors (Eng et al., 2001; Banikazemi et al., 2007) and development of IgG antibodies to agalsidase (Eng et al., 2001; Schiffmann et al., 2001). Pa-

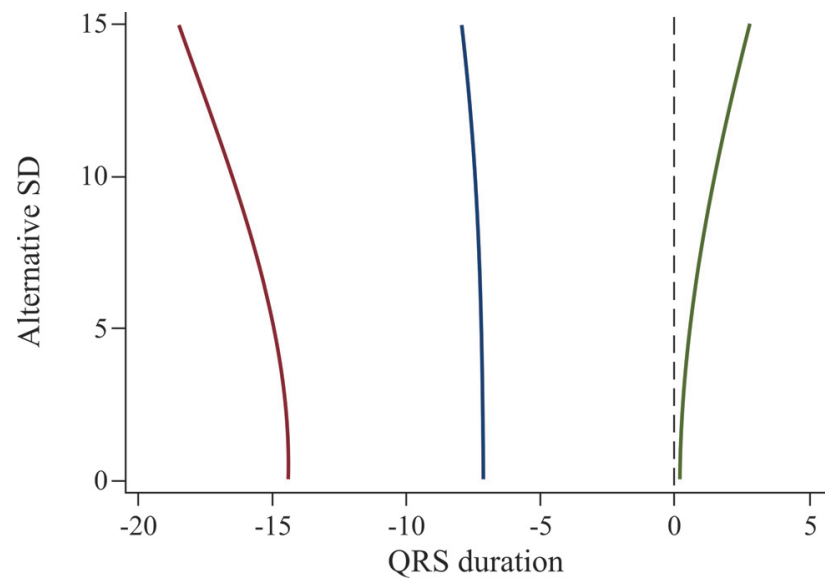

Figure 1 - Summary results under alternative values for the standard deviation for the study by Hughes et al. (2008). The blue line depicts the summary, unstandardized mean difference, whereas red and green lines denote lower and upper 95\% confidence limits, respectively. Dashed line represents the null effect. Results are based on a random effects model. tients given ERT were at increased odds for fever (OR, 5.2; 95\% CI, 2.1 to 12.8) (Figure 2) and rigors (OR, 6.68; $95 \% \mathrm{IC}, 3.04$ to 14.64$)$ when compared to placebo. The risk of hypertension did not reach statistical significance (OR, $3.01 ; 95 \%$ CI, 0.9 to 10.1). The Cochran Q test and the $I^{2}$ statistic showed low statistical heterogeneity between these two studies (for fever $\mathrm{Q}, 0.01 ; I^{2}, 0 \%$, for rigor $\mathrm{Q}, 0.11 ; \mathrm{I}^{2}$ $0 \% \mathrm{~s}$ and for hypertension $\mathrm{Q}, 0.49 ; I^{2}, 0 \%$ ). Development of IgG antibodies occurred in 3/14 patients treated with agalsidase at a dose of $0.2 \mathrm{mg} / \mathrm{kg} /$ infusion in the study by Schiffmann et al. (2001) and in 3/7 patients in the study by Hughes et al. (2008). In patients receiving $1 \mathrm{mg} / \mathrm{kg} / \mathrm{infu}-$ sion, the development of antibodies was described only by Eng et al. (2001), and included the open-label phase, with $51 / 58$ patients seroconverted.

It was possible to perform the metanalysis with the patients evaluated in the double-blind phase by Schiffmann et al. (2001) and Hughes et al. (2008). As expected, there were increased odds for development of IgG to agalsidase (OR, 9.96; 95\% CI, 1.81 to 54.80), without heterogeneity between these two studies (Q, $\left.0.05 ; \mathrm{I}^{2} 0 \%\right)$. However, this immune reaction did not appear to interfere with the efficacy or safety of treatment and did not correlate with the incidence of infusion-related adverse events (Eng et al., 2001; Schiffmann et al. 2001).

\section{Discussion}

This SR/meta-analysis, designed as a critical assessment of the available literature on ERT for FD, was intended to establish the efficacy and safety of this intervention. Our results are in agreement with previously published SRs on this issue (Connock et al., 2006; Lidove et al., 2007; Schaefer et al., 2009; El Dib and Pastores, 2010), although the search strategies and the inclusion criteria were different. Besides that, this is the first paper to present its results in form of a meta-analysis.

Unfortunately, the available RCTs exhibited substantial heterogeneity in endpoints, with the latter ranging from

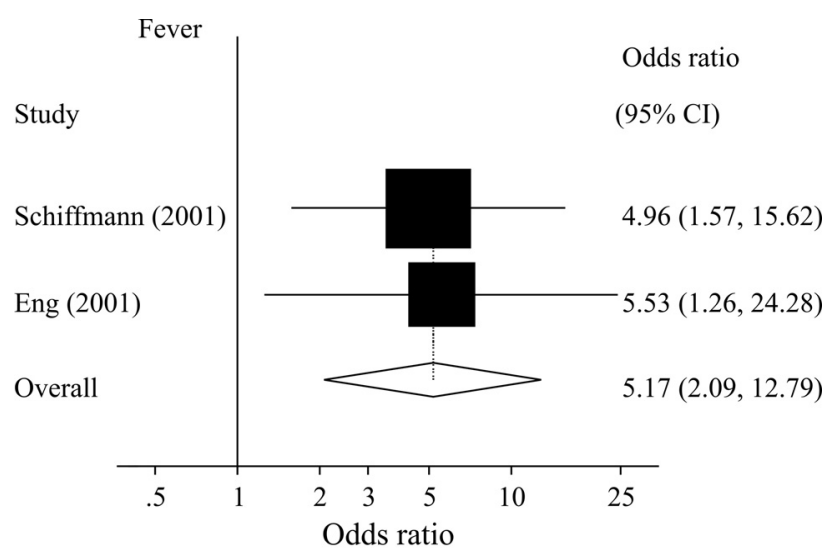

Figure 2 - Meta-analysis: increased odds of fever as an adverse event of enzyme replacement therapy (ERT) as compared to placebo. 
surrogate endpoints such as tissue deposition of GL-3, to major clinical events such as stroke and death. This heterogeneity posed a major challenge to the comparisons and prevented the collection of sufficient data for a metaanalysis of all relevant variables. Furthermore, patients were enrolled at different phases of the disease, which may have influenced their response to ERT. Another limitation was the very restricted recruitment of female patients and no enrollment of asymptomatic patients or patients under the age of 16, thus making it difficult to extend to these populations any conclusions as to the efficacy and safety of ERT.

Because FD is a rare disease, the number of patients available for clinical trials is rather limited, and this restricts the statistical power of any assessment of relevant endpoints. The guiding principle behind treatment of diseases of exceedingly low incidence often referred to as "orphan diseases," runs into bioethical issues involving questions such as equity, scarcity of resources, and the legal concept of the "proviso of possibility" (Vorbehalt des Möglichen). This broad set of challenges means that orphan diseases and the agents meant to treat them - known as orphan drugs - would require specific ways of assessing efficacy and safety. In the case of FD, the methodological limitation of available RCTs creates difficulties in the decision making process not only for drug approval but also for reimbursement. In the author's opinion, the decision about reimbursement for agalsidase should also take into account the fact that there is no other specific treatment for these diseases, as well as the public opinion on this issue.

As of the time of writing, only one published study had assessed clinical endpoints. In this single trial, ERT was not found to reduce mortality or slow progression of kidney, cerebrovascular or heart disease in patients with mild-to-moderate renal impairment, and was not able to reduce the individual or combined incidence of renal, cerebrovascular or cardiovascular events as compared to placebo (Banikazemi et al., 2007). Subgroup analyses showed a greater effect size for treatment in patients with mild kidney disease, which suggests that ERT may be most beneficial in patients with early-stage deterioration of renal function (Banikazemi et al., 2007).

FD is believed to induce neuropathy secondary to GL-3 storage. These changes are considered the main cause of chronic pain in FD patients and are also associated with limb paresthesias, reduced sensitivity to temperature and significant reductions in intraepidermal nerve fiber density. One study tested the hypothesis that intraepidermal nerve fiber density and thermal threshold could be useful markers of central nervous system response to ERT. However, the study showed no significant differences between ERT and placebo for the studied parameters, and there is no evidence that ERT enables nerve regeneration in the epidermis (Schiffmann et al., 2006). Neuropathic pain is usually described as one of the most frequent and debilitating symp- toms of FD, and is rarely amenable to pharmacological treatment. One RCT found improvement in neuropathic pain as measured by the Brief Pain Inventory (Schiffmann et al., 2001). But there are some confounding factors in this study, as methods are unclear as to how neuropathic pain medication was withdrawn prior to pain measurement, and that patients allocated to the placebo group had higher pain scores at baseline. Another study used the McGill Pain Questionnaire and found substantial improvement in ERT and placebo groups alike (Eng et al., 2001). Therefore, further studies of pain intensity, measured with the same instruments, are required before more robust conclusions can be drawn.

ERT was not associated with improved renal function (Schiffmann et al., 2001; Banikazemi et al., 2007) or proteinuria (Banikazemi et al., 2007), changes in the cytoarchitecture of the kidney (Schiffmann et al., 2001), cardiopulmonary stress test performance (Bierer et al., 2006), or diastolic blood pressure (Bierer et al., 2006). A reduction in left ventricular mass was found, but was only detectable on MRI, not on echocardiography (Hughes et al., 2008). All other cardiac-related parameters remained unchanged (Eng et al., 2001; Schiffmann et al., 2001; Hughes et al., 2008). Two studies carried out in the same patient cohort (Moore et al., 2001, 2002) assessed changes in cerebral blood flow at rest and after visual stimuli or acetazolamide challenge and found this parameter to be significantly improved in patients receiving ERT.

Most studies reported reductions in GL-3 buildup in heart, kidney and skin tissue at higher doses and in plasma at both doses (Eng et al., 2001; Schiffmann et al., 2001; Hughes et al., 2008). However, studies that assessed different doses used different methods for measuring GL-3, which makes it difficult to draw any conclusions as to the existence of a dose-dependent effect of agalsidase. Furthermore, although GL-3 represents the biochemical manifestation of FD in body tissues, the reduction in GL-3 buildup is of uncertain clinical significance.

Only one RCT evaluated quality of life (as measured by the SF-36 instrument). However, results were described qualitatively and reported as "improvement" in both groups, and no description of questionnaire administration methods was provided (Eng et al., 2001).

Overall, ERT was well tolerated. Mild and severe adverse effects were rare, and any that did occur were easily controlled with premedication and slower infusion rates (Eng et al., 2001; Schiffmann et al., 2001; Banikazemi et al., 2007). Although not all studies approached the issue of adverse events or development of IgG antibodies, adverse events appear to be more common in patients receiving higher doses of agalsidase. On the other hand, the lowerdose study may simply have been underpowered to assess these effects (Eng et al., 2001), as the higher-dose study enrolled a larger patient sample and featured longer follow-up (Schiffmann et al., 2001; Banikazemi et al., 2007). 
Very few variables could be included in the metaanalysis, due to little overlap of endpoints and results in the available studies. According to our data, ERT was not significantly associated with changes in QRS duration or development of hypertension as a treatment-emergent adverse effect. Not surprisingly, the meta-analysis showed that patients given ERT are at a higher risk of fever, rigors and development of $\operatorname{IgG}$ antibodies to agalsidase during infusion when compared to the placebo group.

In conclusion, although the RCTs carried out thus far have failed to elucidate all questions concerning the efficacy of ERT, this therapy appears to provide some benefit in FD. The current evidence suggests that ERT with agalsidase reduces the severity of neuropathic pain, improves pain-related quality of life, brings about minor changes in left ventricular mass, and reduces plasma, urine and tissue levels of GL-3. The available evidence also suggests that ERT is able to slow down the progression of FD. However, further studies with greater methodological quality, larger sample sizes and better assessment of clinical endpoints are required before firm conclusions can be drawn.

\section{Acknowledgments}

This study was supported by the CNPq and the Brazilian Ministry of Health through $\mathrm{MCT} / \mathrm{CNPq} / \mathrm{MS}$ SCTIE-DECIT grant no. 033/2007. The authors would like to thank CAPES for PhD grants awarded to FV and TA.

The authors would like to thank Tiago V. Pereira for assistance with statistical analysis and for helpful comments on earlier versions of this article.

\section{References}

Banikazemi M, Bultas J, Waldek S, Wilcox WR, Whitley CB, McDonald M, Finkel R, Packman S, Bichet DG, Warnock DG, et al. (2007) Agalsidase-beta therapy for advanced Fabry disease: A randomized trial. Ann Intern Med 146:7786.

Bierer G, Balfe D, Wilcox WR and Mosenifar Z (2006) Improvement in serial cardiopulmonary exercise testing following enzyme replacement therapy in Fabry disease. J Inherit Metab Dis 29:572-579.

Blom D, Speijer D, Linthorst GE, Donker-Koopman WG, Strijland A and Aerts JM (2003) Recombinant enzyme therapy for Fabry disease: Absence of editing of human alpha-galactosidase A mRNA. Am J Hum Genet 72:23-31.

Bradburn MJ, Deeks JJ, Berlin JA and Russell Localio A (2007) Much ado about nothing: A comparison of the performance of meta-analytical methods with rare events. Stat Med 15:26:53-77.

Connock M, Juarez-Garcia A, Frew E, Mans A, Dretzke J, FrySmith A and Moore D (2006) A systematic review of the clinical effectiveness and cost-effectiveness of enzyme replacement therapies for Fabry's disease and mucopolysaccharidosis type 1. Health Technol Assess 10:iii-iv, ix-113.

Desnick RJ, Ioannou YA and Eng CM (2001) Alpha-galactosidase A deficiency: Fabry disease. In: Scriver CR, Beau- $\operatorname{det}$ AL, Sly WS, Valle D, Kinzler KE and Vogelstein B (eds) The Metabolic and Molecular Bases of Inherited Diseases. 8th edition. McGraw-Hill, New York, pp 3733-3774.

DerSimonian R and Laird N (1986) Meta-analysis in clinical trials. Control Clin Trials 7:177-188.

El Dib RP and Pastores GM (2010) Enzyme replacement therapy for Anderson-Fabry disease. Cochrane Database Syst Rev CD006663.

Eng CM, Guffon N, Wilcox WR, Germain DP, Lee P, Waldek S, Caplan L, Linthorst GE and Desnick RJ (2001) Safety and efficacy of recombinant human alpha-galactosidase A replacement therapy in Fabry's disease. NEngl J Med 345:916.

Eng CM, Fletcher J, Wilcox WR, Waldek S, Scott CR, Sillence DO, Breunig F, Charrow J, Germain DP, Nicholls K, et al. (2007) Fabry disease: Baseline medical characteristics of a cohort of 1765 males and females in the Fabry Registry. J Inherit Metab Dis 30:184-192.

Hughes DA, Elliott PM, Shah J, Zuckerman J, Coghlan G, Brookes J and Mehta AB (2008) Effects of enzyme replacement therapy on the cardiomyopathy of Anderson-Fabry disease: A randomised, double-blind, placebo-controlled clinical trial of agalsidase alfa. Heart 94:153-158.

Lee K, Jin X, Zhang K, Copertino L, Andrews L, Baker-Malcolm J, Geagan L, Qiu H, Seiger K, Barngrover D, et al. (2003) A biochemical and pharmacological comparison of enzyme replacement therapies for the glycolipid storage disorder Fabry disease. Glycobiology 13:305-313.

Lidove O, Joly D, Barbey F, Bekri S, Alexandra JF, Peigne V, Jaussaud R and Papo T (2007) Clinical results of enzyme replacement therapy in Fabry disease: A comprehensive review of literature. Int J Clin Pract 61:293-302.

Meikle PJ, Hopwood JJ, Clague AE and Carey WF (1999) Prevalence of lysosomal storage disorders. JAMA 281:249-254.

Moore DF, Scott LT, Gladwin MT, Altarescu G, Kaneski C, Suzuki K, Pease-Fye M, Ferri R, Brady RO, Herscovitch P, et al. (2001) Regional cerebral hyperperfusion and nitric oxide pathway dysregulation in Fabry disease: Reversal by enzyme replacement therapy. Circulation 104:1506-1512.

Moore DF, Altarescu G, Ling GS, Jeffries N, Frei KP, Weibel T, Charria-Ortiz G, Ferri R, Arai AE, Brady RO, et al. (2002) Elevated cerebral blood flow velocities in Fabry disease with reversal after enzyme replacement. Stroke 33:525-531.

Pieroni M, Chimenti C, De Cobelli F, Morgante E, Del Maschio A, Gaudio C, Russo MA and Frustaci A (2006) Fabry's disease cardiomyopathy: Echocardiographic detection of endomyocardial glycosphingolipid compartmentalization. J Am Coll Cardiol 47:1663-1671.

Pinto LL, Vieira TA, Giugliani R and Schwartz IV (2010) Expression of the disease on female carriers of X-linked lysosomal disorders: A brief review. Orphanet J Rare Dis 5:14.

Politei JM and Capizzano AA (2006) Magnetic resonance image findings in 5 young patients with Fabry disease. Neurologist 12:103-105.

Sakuraba H, Murata-Ohsawa M, Kawashima I, Tajima Y, Kotani M, Ohshima T, Chiba Y, Takashiba M, Jigami Y, Fukushige $\mathrm{T}$, et al. (2005) Comparison of the effects of agalsidase alfa and agalsidase beta on cultured human Fabry fibroblasts and Fabry mice. J Hum Genet 51:180-188. 
Schaefer RM, Tylki-Szymanska A and Hilz MJ (2009) Enzyme replacement therapy for Fabry disease: A systematic review of available evidence. Drugs 69:2179-2205.

Schiffmann R, Kopp JB, Austin 3rd HA, Sabnis S, Moore DF, Weibel T, Balow JE and Brady RO (2001) Enzyme replacement therapy in Fabry disease: A randomized controlled trial. JAMA 285:2743-2749.

Schiffmann R, Hauer P, Freeman B, Ries M, Scott LJ, Polydefkis M, Brady RO, McArthur JC and Wagner K (2006) Enzyme replacement therapy and intraepidermal innervation density in Fabry disease. Muscle Nerve 34:53-56.

Spada M, Pagliardini S, Yasuda M, Tukel T, Thiagarajan G, Sakuraba H, Ponzone A and Desnick RJ (2006) High incidence of later-onset fabry disease revealed by newborn screening. Am J Hum Genet 79:31-40.

Thurberg BL, Rennke H, Colvin RB, Dikman S, Gordon RE, Collins AB, Desnick RJ and O'Callaghan M (2002) Globotriaosylceramide accumulation in the Fabry kidney is cleared from multiple cell types after enzyme replacement therapy. Kidney Int 62:1933-1946.

Thurberg BL, Randolph Byers H, Granter SR, Phelps RG, Gordon RE and O'Callaghan M (2004) Monitoring the 3-year efficacy of enzyme replacement therapy in Fabry disease by repeated skin biopsies. J Invest Dermatol 122:900-908.

Vedder AC, Linthorst GE, Houge G, Groener JE, Ormel EE, Bouma BJ, Aerts JM, Hirth A and Hollak CE (2007) Treatment of Fabry disease: Outcome of a comparative trial with agalsidase alfa or beta at a dose of $0.2 \mathrm{mg} / \mathrm{kg}$. PLoS One 2:e598.

Wilcox WR, Oliveira JP, Hopkin RJ, Ortiz A, Banikazemi M, Feldt-Rasmussen U, Sims K, Waldek S, Pastores GM, Lee $\mathrm{P}$, et al. (2008) Females with Fabry disease frequently have major organ involvement: Lessons from the Fabry Registry. Mol Genet Metab 93:112-128.
Woodroffe R, Yao GL, Meads C, Bayliss S, Ready A, Raftery J and Taylor RS (2005) Clinical and cost-effectiveness of newer immunosuppressive regimens in renal transplantation: A systematic review and modelling study. Health Technol Assess 9:1-179, iii-iv.

Wraith JE. 2004. Clinical aspects and diagnosis. In: Platt FM and Walkley SU (eds) Lysosomal Disorders of the Brain. Oxford University Press, Oxford, pp 50-80.

\section{Internet Resources}

Higgins JP and Green S (2011) Cochrane Handbook for Systematic Reviews of Interventions, http://www.cochrane-handbook.org/ (July 1, 2011).

Agência Nacional de Vigilância Sanitária (ANVISA), http://www.anvisa.gov.br (July 1, 2011).

European Agency for the Evaluation of Medicinal Products. European Public Assessment Report for Fabrazyme (2008), http://www.ema.europa.eu/humandocs/Humans/EPAR/fabrazyme/fabrazyme.htm. (July 1, 2011).

European Agency for the Evaluation of Medicinal Products. European Public Assessment Report for Replagal (2007), http://www.ema.europa.eu/humandocs/Humans/EPAR/replagal/replagal.htm (July 1, 2011).

European Medicines Agency (EMEA), http://www.ema.europa.eu/ema/index.jsp?curl = /pages/home/Home_Page.jsp\&jsenabled $=$ true $($ July 1 , 2011).

Food and Drug Administration (FDA), http://www.fda.gov/default.htm (July 1, 2011).

License information: This is an open-access article distributed under the terms of the Creative Commons Attribution License, which permits unrestricted use, distribution, and reproduction in any medium, provided the original work is properly cited. 